\title{
Electronic Transport in Metal Nanocrystal Arrays: The Effect of Structural Disorder on Scaling Behavior
}

\author{
Raghuveer Parthasarathy, Xiao-Min Lin, and Heinrich M. Jaeger \\ James Franck Institute and Department of Physics, University of Chicago, Chicago, IL 60637
}

(October 25, 2018)

\begin{abstract}
We investigate the impact of structural disorder on electronic transport in gold nanocrystal monolayers. Arrays ranging from void-filled networks to well-ordered superlattices show clear voltage thresholds $\left(V_{\mathrm{T}}\right)$ due to Coulomb blockade, and temperature-independent conduction indicative of quantum tunneling. Current-voltage characteristics of arrays with and without long-range structural order were found to collapse onto distinct scaling curves. The former follow a single power-law: $I \sim\left(V-V_{\mathrm{T}}\right)^{\zeta}, \zeta=2.25 \pm 0.1$. The latter show additional structure, reflecting the underlying disordered topology.
\end{abstract}

The intriguing electronic and optical properties of individual nanocrystal quantum dots have unleashed a flood of interest [1]. However, despite such discoveries as metal-insulator-type transitions in squeezed nanocrystal monolayers [2] and spin-dependent transport in magnetic particle assemblies [3], the simplest nanoparticle array - a single layer of metal nanocrystals - has remained poorly understood. The main reason is that the transport characteristics are strongly affected by three types of disorder: global structural disorder in the array topology, local structural disorder in the interparticle couplings, and local charge disorder due to random, immobile charges in the underlying substrate. Theoretical approaches investigating tunneling transport so far have concentrated on local charge disorder only [4]. A full treatment of the combined types of disorder is not available, even though large differences between spatially ordered and disordered structures might be expected due to the sensitivity of percolative charge transport phenomena to array topology and the exponential dependence of local tunneling resistances on the interparticle spacings. With nanocrystal arrays as "artificial solids" [5] expected to provide useful analogues and tunable testbeds for various bulk correlated electron systems, a full understanding of metal nanocrystal monolayers is vital.

Experimentally, a high degree of structural order has been elusive for arrays between in-plane electrodes, and previous investigations of electrical conduction in $2 \mathrm{D}$ nanocrystal systems have been performed only on small, highly disordered, or multi-layered arrays [3,6 8]. Using newly-developed self-assembly techniques, we have fabricated large highly-ordered monolayers of dodecanethiolligated gold nanocrystals on substrates with in-plane electrodes. Transport measurements and subsequent transmission electron microscopy (TEM) on the same arrays allowed for direct correlation of electronic and structural characteristics. Comparison of these superlattices with void-filled networks for the first time delineates the roles played by the different types of disorder.

Nanocrystals were deposited on silicon substrates coated with $100 \mathrm{~nm}$ amorphous silicon nitride $\left(\mathrm{Si}_{3} \mathrm{~N}_{4}\right)$ (Fig. 1a). Under a $70 \mu \mathrm{m} \times 70 \mu \mathrm{m}$ area, the $\mathrm{Si}$ was etched away to leave a freestanding $\mathrm{Si}_{3} \mathrm{~N}_{4}$ membrane "window," allowing TEM imaging [9]. Thin (20nm) Cr electrodes were patterned using electron-beam lithography. 1-dodecanethiol ligated gold nanocrystals were synthesized as described in Ref. [10] and dissolved in toluene to a concentration of about $2.4 \times 10^{13} \mathrm{ml}^{-1}$. The gold core radii varied from sample to sample $(2.2-2.9 \mathrm{~nm})$, but for each sample were monodisperse to within $5 \%$.

Two different array preparation techniques yielded two distinct classes of arrays: samples with and without large-scale structural disorder. Simple deposition of 15$20 \mu \mathrm{l}$ of colloid onto a substrate produced, upon drying, a nanocrystal monolayer of well-packed regions with shortrange order, coexisting with numerous voids (area fraction 15-20\%) throughout the 2D plane (Fig. 1b). Higher particle concentrations produced an increased amount of multi-layered regions without increasing the long-range order. However, addition of excess dodecanethiol (volume fraction $6.3 \times 10^{-3}$ ) to the solution before deposition increased the nanocrystal mobility on the $\mathrm{Si}_{3} \mathrm{~N}_{4}$ surface and prevented rapid dewetting of the solvent from the substrate. As a result, arrays with significantly smaller void or double layer fraction (about $5 \%$ combined) and excellent long-range periodicity could be self-assembled (Figs. 1c,d) [11]. Electronic properties of a total of 14 arrays were measured (7 prepared without and 7 with excess ligand), with dimensions defined by the electrode separations $(200 \mathrm{~nm}<D<700 \mathrm{~nm})$ and widths (fixed at $2 \mu \mathrm{m})$. The resulting $N \times M$ arrays ranged in length from $N=30$ to 90 particles and were $M \approx 270$ nanocrystals wide.

The samples were cooled, in vacuum, to below $77 \mathrm{~K}$ to avoid parasitic conduction through the substrates. DC current-voltage $(I V)$ characteristics were measured using Keithley 614 electrometers and a voltage source. Control measurements on substrates without monolayers showed no detectable currents $(<0.02 \mathrm{pA})$ up to $\pm 20 \mathrm{~V}$. We imaged each array by TEM after the transport measurements. From analysis of the center-to-center distances, $d$, and the particle radii, $r$, distributions of the interparticle spacings $2 s=d-2 r$ (Fig. 1a) were obtained, resulting in $s=0.85 \pm 0.1 \mathrm{~nm}$ for the arrays with voids. For the well- 


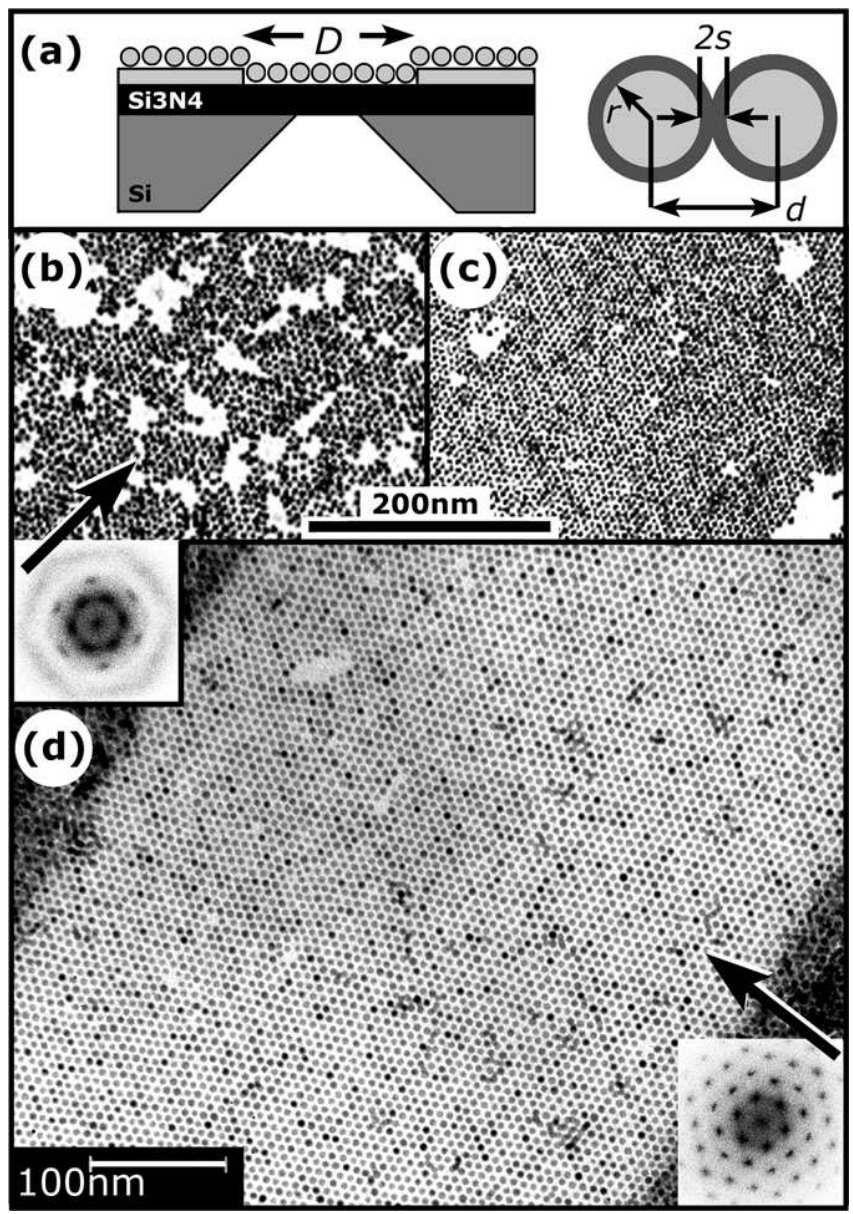

FIG. 1.

(a) Sketch of a nanocrystal monolayer and in-plane electrodes (not to scale), and of the interparticle geometry. (b) TEM image (detail) of a typical array formed without excess dodecanethiol, showing many voids and an absence of long-range order. The 2D Fourier Transform (2DFT) is of a larger area. (c) Typical superlattice formed with excess dodecanethiol, showing $<5 \%$ voids and long-range ordering. (d) Highly ordered superlattice (and 2DFT) between electrodes visible at the upper left and lower right. The random nanocrystal image intensities are due to random Bragg diffraction of the electron beam.

ordered superlattices the excess dodecanethiol increased the mean spacing to $s=1.2 \pm 0.1 \mathrm{~nm}$.

The $I V$ curves (Fig.2) were highly symmetric, of the same overall shape for all samples, and showed no hysteresis at the slow ramp rates used $(<5 \mathrm{mV} / \mathrm{s})$. The strongly non-ohmic behavior, in which current flow requires the applied voltage to exceed a finite threshold, is characteristic of Coulomb blockade of transport [3, [12 14]. The electrostatic energy needed to add one electron to a quantum dot of charge $q$ and (self-) capacitance $C_{0}=4 \pi \epsilon \epsilon_{0} r$ leads to a single-particle Coulomb blockade voltage, $V_{0}=q / C_{0}$, below which tunneling is suppressed (Fig. 2, lower inset). For $V>V_{0}$, current flows with resistance $R=\mathrm{d} V / \mathrm{d} I ; R \gg h / e^{2}$ for our nanocrystals. The overall $I V$ characteristic then

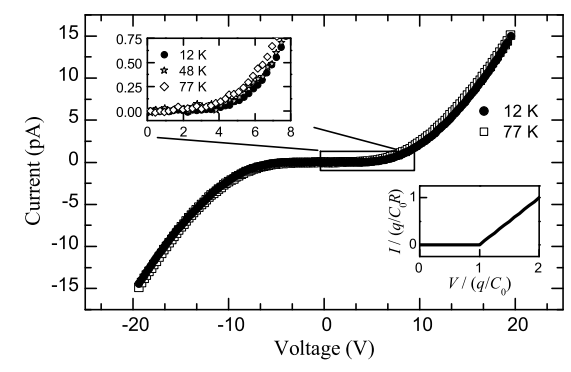

FIG. 2. $I V$ curves for a typical superlattice $(D=330 \mathrm{~nm}$ $\times$ width $2 \mu \mathrm{m})$. The upper inset magnifies the data. The lower inset shows a schematic $I V$ curve for a single nanocrystal, as described in the text.

arises from the series-parallel combination of many tunneling paths from particle to particle throughout the array. Random, parasitic charges in the substrate - local charge disorder - induce the effective charges $q \in(0, e)$ on the nanocrystals, randomly placing the local $V_{0}$ in the interval $\left(0, e / C_{0}\right)$, leaving $R$ unchanged. Local structural disorder, on the other hand, produces variations in the tunnel distance, $2 s$, and thus a wide (most likely $\log$-normal) distribution of $R$, leaving $V_{0}$ essentially unchanged [15]. The exponential dependence of $R$ on $s$ makes a large variance in the interparticle resistances almost a certainty, even in well-assembled arrays (e.g. Fig. 1d).

Using the dielectric constant $\epsilon \approx 2$ for dodecanethiol, and $q=e / 2$, we find $C_{0} \approx 0.5 \mathrm{aF}$ and $V_{0} \approx 150$ $\mathrm{mV}$ for a typical $\mathrm{Au}$ nanocrystal. The capacitance between neighboring particles [16], based on the geometry observed by TEM, is $C_{12}=0.25 \mathrm{aF}<C_{0}$. In this regime of small interparticle capacitive coupling, hysteresis due to long-range charge re-arrangements is not expected 四. Furthermore, for all accessible temperatures $e^{2} / \max \left\{C_{0}, C_{12}\right\} \gg \mathrm{k}_{\mathrm{B}} T$ and thus the array transport properties are essentially temperature-independent (Fig. 2). This behavior implicates direct, interparticle quantum tunneling as the conduction mechanism and is in contrast to the strong temperature dependence observed in other nanocrystal systems with large Coulomb blockade voltages [3]6]. Arrhenius behavior in those systems we believe is due to activated hopping via trapping sites provided by electron-rich $\pi$-bonded linker molecules attached to the metal particle cores. This difference between tunneling and activated hopping suggests that chemical modifications to nanocrystalline systems may change fundamentally the physics governing transport, and highlights two different routes towards control of nanostructure conductance: physical tailoring of separations between metal cores and chemical manipulation of molecular links between the cores.

How charge disorder alone affects the global $I V$ charac- 
teristics measured across large arrays has been calculated by Middleton and Wingreen (MW) [4]. They find a welldefined, global threshold voltage, $V_{\mathrm{T}}$, which delineates a second order phase transition: for $V<V_{\mathrm{T}}$ the array current is zero, and for $V>V_{\mathrm{T}}$ the array conducts with a power-law scaling form, $I \sim M\left(V_{0} / R\right)\left[\left(V-V_{\mathrm{T}}\right) / V_{\mathrm{T}}\right]^{\zeta}$. In Figure 3 we plot the normalized current, $i=I R /\left(M V_{0}\right)$, as a function of normalized voltage above threshold, $v=\left(V-V_{\mathrm{T}}\right) / V_{\mathrm{T}}$, for all 14 measured arrays. The array width $M$ was obtained from the TEM images. $R$ we adjusted to obtain collapse of the curves. Typical $R$ were around 50 and $300 \mathrm{~T} \Omega$ for arrays without and with excess dodecanethiol, respectively, the increase corresponding to the increase in average particle spacing described above. These $R$ are in accord with conducting-tip atomic force microscope studies of tunneling through selfassembled alkanethiol monolayers 17]. Consistent choice of $V_{\mathrm{T}}$ (not far from the values estimated by eye from the $I V$ curves, e.g. Fig. 2) produced a robust scaling behavior of the $I V \mathrm{~s}$ for the ordered arrays assembled with excess ligands (Fig. 3a), yielding an average $\zeta=2.25 \pm 0.1$.

The conductivity exponent $\zeta$ is related to the meandering of current paths in the charge-disordered landscape. Even in regular 2D arrays current paths are not straight, but exhibit transverse fluctuations that extend a distance $\xi_{\perp} \propto v^{-\eta}$ due to the quenched disorder [4]. The total current above threshold across an array of width $M$ is proportional to the number of independent parallel paths, $M / \xi_{\perp}$, which leads to $i \propto v M / \xi_{\perp} \propto M v^{\eta+1}$, i.e. $\zeta=\eta+1$. The determination of the transverse correlation length exponent $\eta$ arises in many physical phenomena connected to interface growth or directed percolation. MW argue that $\eta=1 / z$, where $z=3 / 2$ is the roughness exponent for Kardar-Parisi-Zhang (KPZ) models for interface growth in $2 \mathrm{D}[18$; thus $\zeta \approx 5 / 3$ [19]. Simulations show $\zeta<\approx 2$ (i.e. $\eta<\approx 1$ ) for square arrays of up to $400 \times 400$ particles 4 . The power-law form of the data in Fig. 3a suggests that scaling models are indeed valid, but the measured $\zeta$ is significantly larger than predicted by available theory.

Several previous experiments have reported a wide, sample-dependent spread in the scaling exponent: $1.4<$ $\zeta<2.0$ for lithographically patterned junction arrays [12,13, $1.6<\zeta<2.1$ for polydisperse nanocrystals [8], and $2.2<\zeta<2.7$ for small multi-layered arrays [3]. This contrasts with the highly reproducible values we observe for $\zeta$ in the structurally ordered arrays. However, we find that arrays with large void fraction display $i(v)$ scaling behavior with characteristic slope changes as well as larger sample-to-sample variations (Fig. 3b). The differences between the two classes of IVs may be understood in terms of changes in network topology. At large void fraction, neighboring voids produce bottlenecks, locally cutting off the transverse correlation length $\xi_{\perp}$. In the extreme case, conduction is reduced to several parallel $1 \mathrm{D}$ channels, each with linear $i \propto v$ but threshold $V_{\mathrm{T}}$ dis- tributed over some range. For $V$ barely larger than the smallest $V_{\mathrm{T}}$ only one channel is open and the overall $I V$ is linear. As the applied voltage is increased, there will be a cross-over region in which a growing number of parallel channels conduct. This is born out by the simulated $I V$ characteristic of such a $(1+1) \mathrm{D}$ system (Fig. $3 \mathrm{~b}$, inset). Depending on the number of independent channels, behavior resembling power-laws in the cross-over region with exponents ranging from $\zeta \approx 1$ to $\zeta>2.5$ can easily be reproduced [20]. For sufficiently large $v$ all $1 \mathrm{D}$ channels have opened and the overall $I V$ necessarily becomes linear again. Actual arrays most likely are amalgams of locally 2D patches connected by $1 \mathrm{D}$ channels. Therefore, once all 1D bottlenecks are filled, the overall $I V$ characteristics are dominated by the remaining $2 \mathrm{D}$ patches and $\zeta$ approaches a value close to that of ordered 2D arrays. This is the behavior seen in Fig. $3 \mathrm{~b}$, where $\zeta \approx 2.7$ in the cross-over region turns over to $\zeta=2.16 \pm 0.1$ beyond $v \approx 2$.

Even for our largest accessible $v$, neither the $I V \mathrm{~s}$ of the ordered nor disordered arrays turn linear (Fig. 3a,b), as would be expected once $\xi_{\perp}$ approaches a single lattice spacing. This finding is consistent with results from lithographically patterned arrays below $v \approx 10[12$ and shows a remarkable extent of the scaling regime.

The thresholds $V_{\mathrm{T}}$ obtained from the scaling collapse of the ordered arrays grow linearly with array length, $N$ (Fig. 3c): $V_{\mathrm{T}} /\left(e / C_{0}\right)=\alpha N$ with $\alpha=0.25 \pm 0.02$. Simulations [4] show that $\alpha$ for a given lattice depends only on the capacitive coupling between neighboring particles and decreases as coupling increases; $2 \mathrm{D}$ square arrays in the limit $C_{12} / C_{0} \rightarrow 0$ give $\alpha=0.338$. Due to both the larger coordination number in our hexagonal arrays and the finite $C_{12} / C_{0} \approx 0.4$ we expect $\alpha<0.338$, consistent with the measured value. For arrays with large void fraction, $N$ is poorly defined, leading to strongly sampledependent $V_{\mathrm{T}}(N)$ values.

From our findings, two key results emerge. First, sufficiently large amounts of topological disorder, due to voids in the monolayer, lead to distinct deviations from simple power law behavior in the $I V$ characteristics. In situations where direct imaging is impossible, detailed examination of $I V \mathrm{~s}$, therefore, may provide clues about an array's large scale topology. Second, $I V$ characteristics of long-range ordered arrays are well-fit by a single power law, despite the existence of both charge disorder and an inherent wide distribution of tunnel resistances. We believe this indicates that while $R$ may be exponentially sensitive to variations in the interparticle separation, charge disorder nevertheless plays the dominant role in selecting optimal current paths across the array. The reason most likely lies in the extremely non-linear, local $I V$ characteristics (Fig. 2, inset), which effectively shut off all current flow unless $V_{0}$ is exceeded [21]. Within this picture, our finding of an exponent $\zeta \approx 2.25$ in the structurally well-ordered arrays implies $\eta \approx 1.15$ and thus, as 


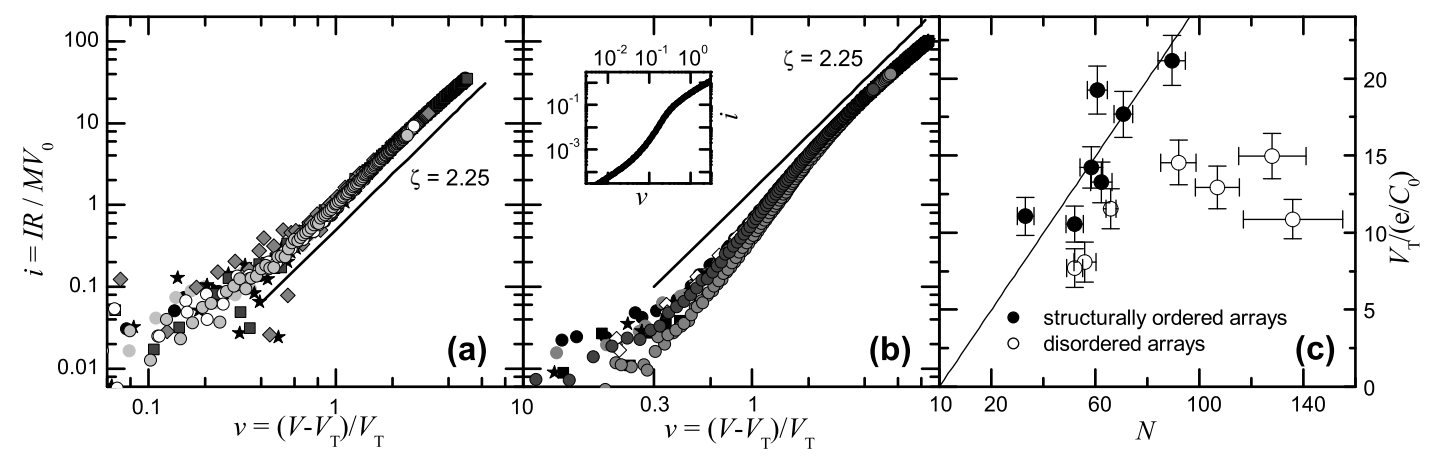

FIG. 3. Scaling behavior of $I V$ curves. (a,b) Log-log plots of all data with normalized voltage beyond threshold $v=\left(V-V_{\mathrm{T}}\right) / V_{\mathrm{T}}$ and current $i=I R /\left(M V_{0}\right)$ as described in the text. (a) Data from seven monolayers with long-range structural order. The solid line shows best-fit power-law $i=v^{\zeta}$ with $\zeta=2.25$. (b) Data from seven disordered monolayers. The solid line shows $\zeta=2.25$ as in (a). Inset: $I V$ curve from a $(1+1) \mathrm{D}$ simulation of 100 independent parallel channels each 100 particles in length. (c) Threshold voltage $V_{\mathrm{T}}$, in units of $e / C_{0}$, versus array length $N$. The line is a fit to $V_{\mathrm{T}}=\alpha N\left(e / C_{0}\right)$, which gives $\alpha=0.25$ for the superlattices formed with excess dodecanethiol.

$V_{\mathrm{T}}$ is approached from above, a stronger divergence of $\xi_{\perp}$ than would be expected from charge disorder alone (where $\eta \approx 0.67$ ). In other words, the spread in tunnel resistances appears to produce a more rapid growth of transverse fluctuations in the meandering current paths.

We thank P. Guyot-Sionnest, L. P. Kadanoff, and T. F. Rosenbaum for helpful discussions, and N. W. Mueggenburg and A. W. Smith for experimental assistance. This work was supported by the Keck Foundation through Grant Number 991705, and by the MRSEC program of the National Science Foundation under Award Number DMR-9808595. One of us (RP) acknowledges an NSF Graduate Fellowship, and a Grainger Graduate Fellowship.

[1] G. L. Timp, ed., Nanotechnology (Springer-Verlag, New York, 1999).

[2] C. P. Collier, R. J. Saykally, J. J. Shiang, S. E. Henrichs, and J. R. Heath, Science 277, 1978 (1997).

[3] C. T. Black, C. B. Murray, R. L. Sandstrom, and S. Sun, Science 290, 1131 (2000).

[4] A. A. Middleton and N. S. Wingreen, Phys. Rev. Lett. 71, 3198 (1993).

[5] C. P. Collier, T. Vossmeyer, and J. R. Heath, Annu. Rev. Phys. Chem. 49, 371 (1998).

[6] R. P. Andres et al., Science 273, 1690 (1996)

[7] A. S. Cordan et al., J. Appl. Phys. 87, 345 (2000); C. Vieu et al., J. Vac. Sci. Technol. B 16, 3789 (1998); W. Chen et al., Appl. Phys. Lett. 66, 3383 (1995).
[8] M. N. Wybourne et al., Jpn. J. Appl. Phys. 36, 7796 (1997).

[9] T. L. Morkved et al., Polymer 39, 3871 (1998).

[10] X. M. Lin et al. J. Nanoparticle Res. 2, 157 (2000).

[11] X. M. Lin, H. M. Jaeger, C. M. Sorensen, and K. J. Klabunde (to be published). Without electrodes present, superlattices routinely exhibit long-range order over microns. Electrodes perturb the monolayer formation, leading to variability in superlattice quality.

[12] Ç. Kurdak et al., Phys. Rev. B 57, R6842 (1998).

[13] A. J. Rimberg et al., Phys. Rev. Lett. 74, 4714 (1995); C. I. Duruöz et al., Phys. Rev. Lett. 74, 3237 (1995).

[14] A. Bezryadin et al., Appl. Phys. Lett. 74, 2699 (1999).

[15] In the limit $C_{12}<C_{0}$ the effects of changes in $s$ on $C_{12}$ and $V_{0}$, on $R$.

[16] W. R. Smythe, Static and Dynamic Electricity (McGrawHill, New York, 1950).

[17] D. J. Wold and C. D. Frisbie, J. Am. Chem. Soc. 122, 1970 (2000).

[18] M. Kardar, G. Parisi, and Y.-C. Zhang, Phys. Rev. Lett. 56, 889 (1986); S. Roux et al., J. Phys. A 24, L295 (1991). H. Hinrichsen, Braz. J. Phys. 30, 69 (2000), also cond-mat/9910284.

[19] The KPZ interface roughness exponent $z$ is the ratio of exponents for fluctuations parallel $\left(\nu_{\|}\right)$and perpendicular $\left(\nu_{\perp}\right)$ to the growth direction. Intriguingly, linking $\eta$ to transverse fluctuations only, i.e. $\eta=\nu_{\perp}$ rather than $\eta=$ $1 / z$, and using $\nu_{\perp} \approx 1$ 18 gives $\zeta \approx 2$.

[20] For example, for 100 parallel channels of 100 particles each, $\zeta=2.7$ at the center of the crossover region.

[21] Current can only flow through paths for which $V>$ $\Sigma q_{i} / C_{0}$, irrespective of whether such paths minimize the overall resistance across the array. 